\title{
Minimum number of wavelengths equals load in a DAG without internal cycle
}

\author{
Jean-Claude Bermond, and Michel Cosnard \\ Mascotte Project, I3S(CNRS/UNSA)/INRIA \\ 2004 route des Lucioles - B.P. 93 \\ F-06902 Sophia-Antipolis Cedex - FRANCE \\ \{jean-claude.bermond,michel.cosnard\}@inria.fr
}

\begin{abstract}
Let $\mathscr{P}$ be a family of dipaths. The load of an arc is the number of dipaths containing this arc. Let $\pi(G, \mathscr{P})$ be the maximum of the load of all the arcs and let $w(G, \mathscr{P})$ be the minimum number of wavelengths (colors) needed to color the family of dipaths $\mathscr{P}$ in such a way that two dipaths with the same wavelength are arc-disjoint.

Let $G$ be a DAG (Directed Acyclic Graph). An internal cycle is an oriented cycle such that all the vertices have at least one predecessor and one successor in $G$ (said otherwise every cycle contain neither a source nor a sink of $G$ ). Here we prove that if $G$ is a DAG without internal cycle, then for any family of dipaths $\mathscr{P}, w(G, \mathscr{P})=\pi(G, \mathscr{P})$. On the opposite we give examples of DAGs with internal cycles such that the ratio between $w(G, \mathscr{P})$ and $\pi(G, \mathscr{P})$ cannot be bounded.

We also consider an apparently new class of DAGs, which is of interest in itself, those for which there is at most one dipath from a vertex to another. We call these digraphs UPP-DAGs. For these UPP-DAGs we show that the load is equal to the maximum size of a clique of the conflict graph. We show that if an UPP-DAG has only one internal cycle, then for any family of dipaths $w(G, \mathscr{P})=$ $\left\lceil\frac{4}{3} \pi(G, \mathscr{P})\right\rceil$ and we exhibit an UPP-DAG and a family of dipaths reaching the bound. We conjecture that the ratio between $w(G, \mathscr{P})$ and $\pi(G, \mathscr{P})$ cannot be bounded.
\end{abstract}

\section{Introduction}

The problem we consider is motivated by routing, wavelength assignment and grooming in optical networks. But it

$\overline{1-4244-0910-1 / 07 / \$ 20.00 ~ @ 2007 ~ I E E E . ~}$ can be of interest for other applications in parallel computing, where the graph will represent for example the precedence graph of a program or for scheduling complex operations on pipelined operators. A generic problem in the design of optical networks, [11, 13]), consists of satisfying a family of requests (or a traffic matrix) under various constraints like capacity constraints. The optimization problem associated consists in designing, for a given family of requests, a network optimizing some criteria, such as minimizing the number of wavelengths or the number of ADMs (Add Drop Multiplexers).

A request is satisfied by assigning to it a dipath in the network. A family of requests is satisfied, if we can route them in such a way that the capacity constraints of the network are satisfied. This is known as the routing problem. For a given routing let us define the load of an arc as the number of routes (dipaths) containing it and the load of the routing as the maximum load of the arcs. Typically one wants either to insure that the load of an arc does not exceed the capacity of this arc or to minimize the load of a routing satisfying a given family of requests.

Many backbone networks are now WDM optical ones. Indeed wavelength division multiplexing (WDM) enables to use the bandwidth of an optical fiber by dividing it in multiple non overlapping frequencies or wavelength channels. Satisfying a request in a WDM optical network consists in assigning to it a route (dipath), but also a wavelength, which shall stay unchanged if no conversion is allowed. Therefore the constraint is now that two requests, having the same wavelength, have to be routed by two arc disjoint dipaths or, equivalently, two requests whose associated dipaths share an arc, have to be assigned different wavelengths. Hence the scarce resource is the number of available wavelengths. For a given traffic matrix, either one wants to insure that the family of requests can be satisfied with the available num- 
ber of wavelengths or one wants to minimize the number of wavelengths used. This problem is known in the literature as the RWA (Routing and Wavelength Assignment) problem.

Note that requests are satisfied on a virtual (logical) network which is itself embedded in the physical network (in fact there might be many layers). It is the case for example when considering SONET/WDM rings or in MPLS over WDM networks; in the latter case the RWA problem has to be considered for the lightpaths [6, 7]. Anyway, at the conceptual level of modeling of this article, the problems are the same and we will use the word request to indicate a connection at the upper level.

Minimizing the load or/and the number of wavelengths is a difficult problem and in general an NP-hard problem. These problems have been extensively studied in the literature for various topologies or special families of requests like multicast or all-to-all (see for example the survey [1] or $[10,12])$. Many particular cases where the minimum number of wavelengths is equal to the minimum routing load have been given. For example, in [2] it is shown that for any digraph and for a multicast instance (all the requests have the same origin), there is equality and both problems can be solved in polynomial time. For some topologies the load might be easily computed, but the minimum number of wavelengths is NP-hard to compute as it is related to coloring problems. This is the case for symmetric trees (see the survey [5]). However, for symmetric trees it has been proved that there is equality for the all to all instance ([9]) and approximation algorithms have been given ([5, 8]).

As the RWA problem is very difficult to solve, it is often split into two separate problems. First one solves the routing problem by determining dipaths which minimize the load or are easy to compute like shortest paths. Then, the routing being given, the wavelength assignment problem is solved. In that case the input of the problem is not a family of requests but a family of dipaths $\mathscr{P}$. We will denote by $\pi(G, \mathscr{P})$ the maximum of the load of all the arcs of the digraph $G$ for the family $\mathscr{P}$. Determining the minimum number $w(G, \mathscr{P})$ of wavelengths (colors) needed to color a family of dipaths $\mathscr{P}$ in such a way that two dipaths with the same wavelength are arc-disjoint is still NP-hard in that case. Indeed it corresponds to finding the chromatic number of the conflict graph whose vertices represent the dipaths and where two vertices are joined if the corresponding dipaths intersect.

There are examples of topologies where there are at most 2 dipaths using an $\operatorname{arc}(\pi(G, \mathscr{P})=2)$, but where we need as many wavelengths as we want (see Figure 1 for the example for 4 wavelengths) and so for these digraphs there is no ratio between $w(G, \mathscr{P})$ and $\pi(G, \mathscr{P})$. In the example we consider $k$ dipaths from $s_{i}$ to $t_{i}$. The dipaths starts in $s_{i}$, then go alternatively right and down till they arrive at the bottom where they go right and up till they arrive at the destination $t_{i}$. Any two dipaths intersect so the conflict graph is complete and we need $k$ colors. However the load of an arc is at most 2 .

Note that, if the original digraph has the property that for any request $(x, y)$ there is a unique dipath from $x$ to $y$, then it is equivalent to consider a family of requests or a family of dipaths.

Here we consider the class of Directed Acyclic Graphs, DAGs, which plays a central role in Parallel and Distributed Computing. Part of our motivation came when we tried to extend the results obtained in [4] for paths motivated by grooming problems for the paths $([3,7])$. In fact, we first proved that for rooted trees (directed trees where there is a unique dipath from the root to any vertex), for any family of requests, the minimum number of wavelengths is equal to the load.

The example given above in Figure 1 being a DAG there is no hope to bound ratio between $w(G, \mathscr{P})$ and $\pi(G, \mathscr{P})$. In this paper we fully characterize when $w(G, \mathscr{P})=$ $\pi(G, \mathscr{P})$ for a DAG. In fact the necessary and sufficient condition is that $G$ does not contain what we call an internal cycle, i.e. an oriented cycle, such that all the vertices have at least one predecessor and one successor in $G$ (said otherwise all cycles contain neither a source nor a sink).

We also consider an apparently new class of DAGs, which is of interest in itself, those for which there is at most one dipath from a vertex to another. We call this property the UPP (Unique diPath Property) and call these digraphs UPP-DAGs. For these UPP-DAGs we show that the load is equal to the maximum size of a clique of the conflict graph. We show that if an UPP-DAG has only one internal cycle, then for any family of dipaths $w(G, \mathscr{P})=$ $\left\lceil\frac{4}{3} \pi(G, \mathscr{P})\right\rceil$ and we exhibit an UPP-DAG and a family of dipaths reaching the bound. We conjecture that the ratio between $w(G, \mathscr{P})$ and $\pi(G, \mathscr{P})$ cannot be bounded.

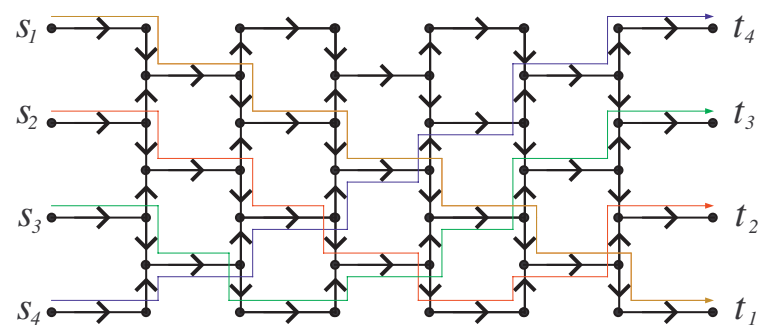

Figure 1. A pathological example 


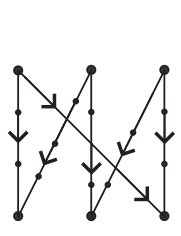

a)

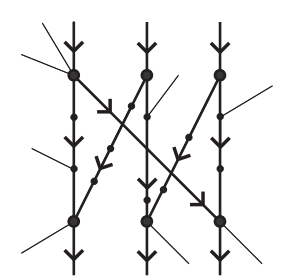

b)
Figure 2. An oriented cycle (a) and an internal cycle (b)

\section{Definitions}

We model the network by a digraph $G$. The outdegree of a vertex $x$ is the number of arcs with initial vertex $x$ (that is the number of vertices $y$ such that $(x, y)$ is an $\operatorname{arc}$ of $G$ ). The indegree of a vertex $x$ is the number of arcs with terminal vertex $x$ (that is the number of vertices $y$ such that $(y, x)$ is an $\operatorname{arc}$ of $G$ ). A source is a vertex with indegree 0 and a sink a vertex with outdegree 0 . A dipath is a sequence of vertices $x_{1}, x_{2}, \ldots, x_{k}$ such that $\left(x_{i}, x_{i+1}\right)$ is an $\operatorname{arc}$ of $G$. If $x_{k}=x_{1}$ the dipath is called a directed cycle.

A DAG (Directed Acyclic Graph) is a digraph with no directed cycle.

An (oriented) cycle in a DAG consists therefore of an even sequence of dipaths $P_{1}, P_{2}, \ldots, P_{2 k}$ alternating in direction (see Figure 2a). The vertices inside the dipaths have indegree and outdegree 1; those where there is a change of orientation have either indegree 2 and outdegree 0 or indegree 0 and outdegree 2 .

An internal cycle of a DAG $G$ is an oriented cycle such that all its vertices have in $G$ an indegree $>0$ and an outdegree $>0$; said otherwise no vertex is a source or a sink. Hence the vertices where there is a change of orientation in the cycle have a predecessor (resp. a successor) in $G$ if they are of indegree 0 (resp. outdegree 0 ) in the cycle (see Figure $2 b)$.

We will say that a DAG has the UP Property (Unique Path Property) if between two vertices there is at most one dipath. A digraph satisfying this property will be called an UPP-DAG.

If $G$ is an UPP-DAG, then any internal cycle contains at least $2 k \geq 4$ vertices where there is a change of orientation. Otherwise it will consist of a dipath from $x$ to $y$ and a reverse dipath from $y$ to $x$ and so there will be two dipaths from $x$ to $y$.

Given a digraph $G$ and a family of dipaths $\mathscr{P}$, the load of an arc $e$ is the number of dipaths of the family contain-

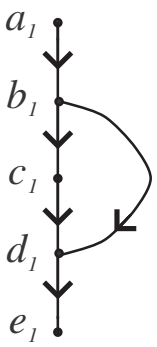

$G$

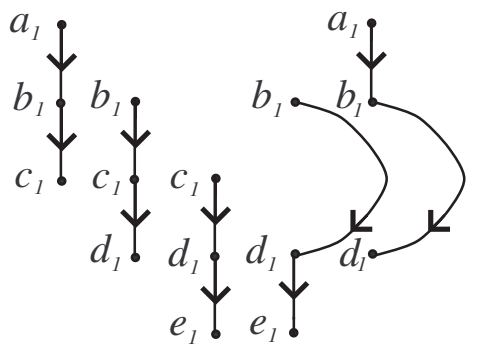

5 paths

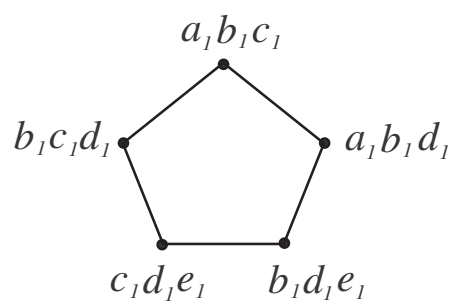

Conflict graph

Figure 3. Example for a DAG with an internal cycle

ing $e$ :

$$
\operatorname{load}(G, \mathscr{P}, e)=|\{P: P \in \mathscr{P} ; e \in P\}|
$$

The load of $G$ for $\mathscr{P}$ will be the maximum over all the arcs of $G$ and we will denote it by $\pi(G, \mathscr{P})$. We will say that two dipaths are in conflict (or intersect) if they share an arc. We will denote by $w(G, \mathscr{P})$ the minimum number of colors needed to color the dipaths of $\mathscr{P}$ in such a way that two dipaths in conflict (sharing an arc) have different colors. Note that $\pi(G, \mathscr{P}) \leq w(G, \mathscr{P})$.

The vertices of the conflict graph associated with $(G, \mathscr{P})$ are the dipaths of $\mathscr{P}$, two vertices being joined if their associated dipaths are in conflict.

Then $w$ is the chromatic number of the conflict graph. Note that $\pi$ is only upper bounded by the clique number of the conflict graph; indeed the $\pi$ dipaths containing an arc $e$ of maximum load are pairwise in conflict. We will show later on, that if $G$ is an UPP-DAG then $\pi$ is exactly the clique number of the conflict graph.

As we have seen in the introduction, there exist DAGs for which there exists a set of dipaths $\mathscr{P}$ such that $\pi(G, \mathscr{P})=$ 2 and $w(G, \mathscr{P})$ is as big as we want. These DAGs have many internal cycles. In Figure 3 , we give an example of a DAG with one internal cycle and a set of 5 dipaths $\mathscr{P}$ 
such that $\pi(G, \mathscr{P})=2$ and $w(G, \mathscr{P})=3$. The dipaths are $a_{1}, b_{1}, c_{1} ; b_{1}, c_{1}, d_{1} ; c_{1}, d_{1}, e_{1} ; b_{1}, d_{1}, e_{1}$ via the second dipath from $b_{1}$ to $d_{1} ; a_{1}, b_{1}, d_{1}$ also via this second dipath. The load is 2 and the conflict graph is a cycle of length 5 and so we need 3 colors to color its vertices. Other examples of family of dipaths $\mathscr{P}$ with $\pi(G, \mathscr{P})=2$ and $w(G, \mathscr{P})=3$ in an UPP-DAG are given in section 4 (Figures 5 and 9).

\section{Main result}

In this section we characterize the DAGs for which for any family $\mathscr{P}$ of dipaths, $w(G, \mathscr{P})=\pi(G, \mathscr{P})$.

Theorem 1 Let $G$ be a DAG without internal cycle. Then, for any family of dipaths $\mathscr{P}, w(G, \mathscr{P})=\pi(G, \mathscr{P})$.

Proof: It is by induction on the number of arcs (the theorem being trivially true if there is no arc or one arc). The idea consists in coloring the arcs of the graph obtained by deleting one arc and shrinking the dipaths containing this arc in such a way that the shrinked dipaths have all different colors and then to extend this coloring to the original graph.

Suppose that the theorem is true for all DAGs with $m$ arcs and let $H$ be a DAG with $m+1$ arcs and $\mathscr{Q}$ be a family of dipaths of $H$. Let $\left(x_{0}, y_{0}\right)$ be an arc of $H$ such that $d^{-} H\left(x_{0}\right)=0\left(x_{0}\right.$ is a source in $\left.H\right)$. Let $G$ be the digraph obtained from $H$ by deleting the arc $\left(x_{0}, y_{0}\right)$ and let $\mathscr{P}$ be the family of dipaths of $G$ obtained from $\mathscr{Q}$ as follows. If $Q$ belongs to $\mathscr{Q}$ and does not contain $\left(x_{0}, y_{0}\right)$, we put $Q$ in $\mathscr{P}$; otherwise if $Q$ contains $\left(x_{0}, y_{0}\right)$ we put in $\mathscr{P}$ the dipath $Q-\left(x_{0}, y_{0}\right)$ obtained by deleting the arc $\left(x_{0}, y_{0}\right)$. In particular, if $Q$ was reduced to the arc $\left(x_{0}, y_{0}\right)$ then we do not consider it.

We have $\pi(G, \mathscr{P}) \leq \pi(H, \mathscr{Q})$. More precisely, $\pi(H, \mathscr{Q})=\max \left(\pi(G, \mathscr{P}) ; \pi_{0}\right)$, where $\pi_{0}$ denotes the load of the $\operatorname{arc}\left(x_{0}, y_{0}\right)$ in $H . \pi_{0}=\operatorname{load}\left(H, \mathscr{Q},\left(x_{0}, y_{0}\right)\right)$.

Let $\mathscr{Q}_{0}$ be the family of dipaths of $\mathscr{Q}$ containing $\left(x_{0}, y_{0}\right)$ and let $\mathscr{P}_{0}$ be the family of non empty dipaths associated with $\mathscr{Q}_{0}$ (obtained by deleting the arc $\left(x_{0}, y_{0}\right)$ ). Note that $\pi_{0}=\left|\mathscr{Q}_{0}\right|$ and therefore $\pi_{0} \geq\left|\mathscr{P}_{0}\right|$ (there are in fact $\pi_{0}$ $\left|\mathscr{P}_{0}\right|$ dipaths in $\mathscr{Q}_{0}$ reduced to the arc $\left.\left(x_{0}, y_{0}\right)\right)$.

By induction hypothesis we can color the family $\mathscr{P}$ of dipaths in $G$ with $\pi(G, \mathscr{P})$ colors. If all the dipaths of $\mathscr{P}_{0}$ have different colors, we can extend the coloring to the family $\mathscr{Q}$ as follows. If a dipath $Q$ is in $\mathscr{Q}_{0}$ and if $P=Q-\left(x_{0}, y_{0}\right)$ is non empty (i.e. $P$ in $\left.\mathscr{P}_{0}\right)$, then color $Q$ with the color of $P$. Finally color the remaining dipaths of $\mathscr{Q}_{0}$, namely the arc $\left(x_{0}, y_{0}\right)$ with the remaining $\pi_{0}-\left|\mathscr{P}_{0}\right|$ colors. The rest of the proof consists in showing that, by appropriate recoloring, we can always find a coloring of the dipaths of $\mathscr{P}$ such all the dipaths of $\mathscr{P}_{0}$ have different colors. Suppose this is not the case and consider a coloring of $\mathscr{P}$ which maximizes the number of colors used by the dipaths of $\mathscr{P}_{0}$. As the colors are not all different, there exists at least one color $\alpha$ used by two different dipaths $P_{0}$ and $P_{1}$ of $\mathscr{P}_{0}$. Among the $\pi(H, \mathscr{Q})$ colors choose a color $\beta$ not used for $\mathscr{P}_{0}$; such a color exists as we have used at most $\left|\mathscr{P}_{0}\right|-1 \leq \pi_{0}-1$ colors for the dipaths of $\mathscr{P}_{0}$.

We will show that we can obtain a valid coloring of the dipaths of $\mathscr{P}$, such that $P_{0}$ keeps its color $\alpha, P_{1}$ gets color $\beta$ and the other dipaths of $\mathscr{P}$ keep their color if it is different from $\alpha$ and $\beta$ and get one of the color $\alpha$ and $\beta$ otherwise (some dipaths initially colored $\alpha$ (resp. $\beta$ ) can be recolored $\beta($ resp. $\alpha)$ ). So, we obtain a valid coloring in which the dipaths of $\mathscr{P}_{0}$ use one color more than in the initial coloring and we get a contradiction with the hypothesis of maximality.

For that purpose, let us change the color $\alpha$ of $P_{1}$ to $\beta$. If the coloring obtained is valid we are done. Otherwise that means that $P_{1}$ intersects some dipaths with color $\beta$; note that these dipaths are not in $\mathscr{P}_{0}$ as color $\beta$ was not used. Recolor with color $\alpha$ the family $\mathscr{P}_{2}$ consisting of all the dipaths of color $\beta$ intersecting $P_{1}$. If we obtain a valid coloring we are done; otherwise that means that some dipaths of $\mathscr{P}$ colored initially $\alpha$ intersect some dipaths of $\mathscr{P}_{2}$. Let $\mathscr{P}_{3}$ be the family of all these dipaths. Then recolor them $\beta$ and so on. If at step $p$, we have recolored a family $\mathscr{P}_{p}$ from color $\gamma$ (where $\gamma$ is $\alpha$ or $\beta$ ) to $\gamma^{\prime}$ (where $\gamma^{\prime}$ is the other color), we recolor at step $p+1$ the family $\mathscr{P}_{p+1}$ of all the dipaths of color $\gamma^{\prime}$ intersecting some dipaths of $\mathscr{P}_{p}$.

We continue the process till we arrive to one of the following situations :

Case A : we have obtained a valid coloring and then our claim is proved

Case B : we have to recolor a dipath already recolored at a preceding step (said otherwise the family $\mathscr{P}_{p}$ and $\mathscr{P}_{q}$ intersect for some indices $p$ and $q$ ).

Case $\mathrm{C}$ : we have to recolor $P_{0}$.

Let us show that case B cannot happen. Indeed suppose at step $p$ we have recolored a dipath $P_{p}$ of color $\gamma$ with color $\gamma^{\prime}$; then suppose at a further step $q$ we want to recolor $P_{p}$. That implies that at step $q-1 P_{p}$, which is at this step of color $\gamma^{\prime}$, intersected a dipath $P_{q-1}$ which has been recolored from color $\gamma$ to $\gamma^{\prime}$. But this implies that in the initial coloring both $P_{p}$ and $P_{q-1}$ were of the same color $\gamma$, contradicting the validity of the initial coloring.

Suppose now case $\mathrm{C}$ happens, then we will exhibit an internal cycle, therefore contradicting the hypothesis that there is no internal cycle and proving the theorem.

Suppose at step $p$ we have to recolor $P_{0}$; this is due to the fact that at step $p-1$ some dipath $P_{p-1}$ has been recolored from color $\beta$ to color $\alpha$ which was due to the existence of a dipath $P_{p-2}$ recolored at step $p-2$ from $\alpha$ to $\beta$ and so on. Thus, there exists a sequence of dipaths $P_{1}, P_{2}, \ldots, P_{p-1}, P_{p}=P_{0}$, alternating in color and such that $P_{i}$ and $P_{i+1}$ intersect in some interval $I_{i}=\left(x_{i}, y_{i}\right)$. 


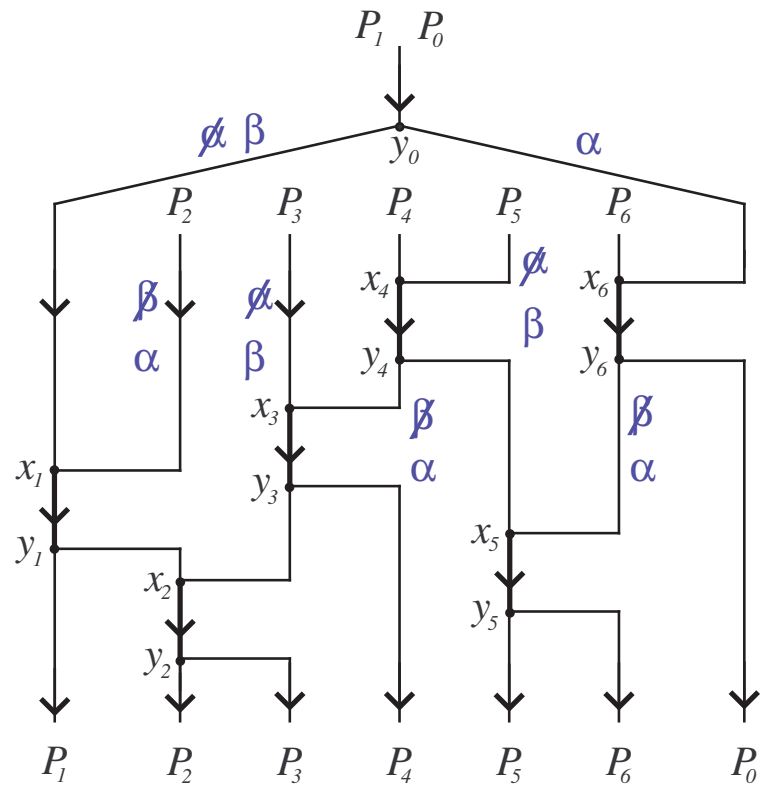

a)

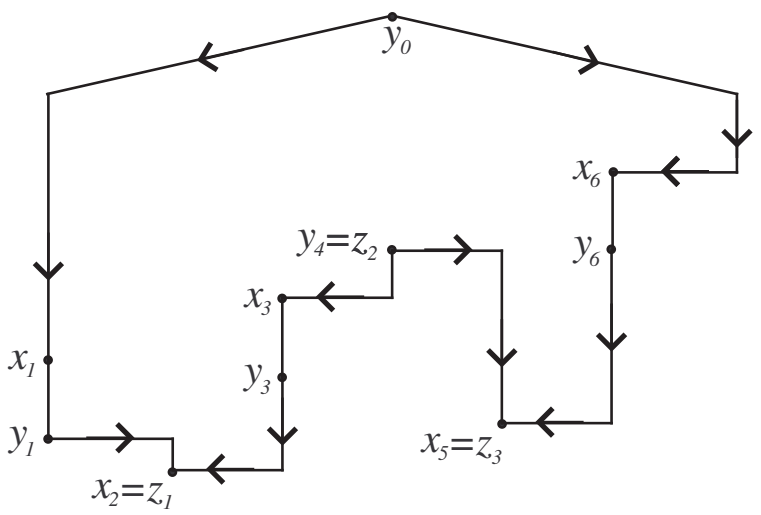

b)

Figure 4. Recoloring in case $\mathrm{C}$

Consider the closed walk

$$
\begin{gathered}
P_{1}\left[y_{0}, x_{1}\right], P_{2}\left[x_{1}, x_{2}\right], \ldots, P_{i}\left[x_{i-1}, x_{i}\right], \ldots \\
\ldots P_{p-1}\left[x_{p-2}, x_{p-1}\right], P_{0}\left[x_{p-1}, y_{0}\right]
\end{gathered}
$$

Some of the paths are directed; some are in the reverse orientation. If $P_{i}\left[x_{i-1}, x_{i}\right]$ is directed, then it does not contain $I_{i}=\left(x_{i}, y_{i}\right)$; otherwise if $P_{i}\left[x_{i-1}, x_{i}\right]$ is in the reverse orientation, then it contains $I_{i}=\left(x_{i}, y_{i}\right)$. Therefore, if $P_{i}\left[x_{i-1}, x_{i}\right]$ is directed and $P_{i+1}\left[x_{i}, x_{i+1}\right]$ is in the reverse orientation the interval $I_{i}=\left(x_{i}, y_{i}\right)$ does not belong to the walk. In contrary, if $P_{i}\left[x_{i-1}, x_{i}\right]$ is in the reverse orientation and $P_{i+1}\left[x_{i}, x_{i+1}\right]$ is directed then both contain $I_{i}=\left(x_{i}, y_{i}\right)$ and in that case we delete the interval from the paths of the walk in order to obtain a simple cycle (without multiple edges). Let this cycle be of the form $C=\left(y_{0}, z_{1}, z_{2}, \ldots, z_{k}, y_{0}\right)$, where $z_{2 h+1}$ corresponds to an $x_{i}$, where $P_{i}\left[x_{i-1}, x_{i}\right]$ is directed and $P_{i+1}\left[x_{i}, x_{i+1}\right]$ is in the reverse orientation and where $z_{2 h+2}$ corresponds to an $y_{i}$, where $P_{i}\left[x_{i-1}, x_{i}\right]$ is in the reverse orientation and $P_{i+1}\left[x_{i}, x_{i+1}\right]$ is directed. $C$ consists of a sequence of directed and in the reverse orientation paths, where all the vertices different from a $z_{j}$ have in and outdegree 1. Vertices $z_{2 h+1}$ have in the cycle indegree 2 and outdegree 0 ; but in $G$ they have outdegree at least 1 , as they are initial vertices of an interval $I_{i}=\left(x_{i}, y_{i}\right)$. Similarly vertices $z_{2 h+2}$ have in the cycle indegree 0 and outdegree 2 ; but in $G$ they have indegree at least 1 , as they are terminal vertices of an interval $I_{i}=\left(x_{i}, y_{i}\right)$. So $C$ is an internal cycle and the theorem is proved.(see Figure 4 for an example).

Theorem 2 If a DAG $G$ contains an internal cycle there exists a set $\mathscr{P}$ of dipaths such that $\pi(G, \mathscr{P})=2$ and $w(G, \mathscr{P})=3$.

Proof: Consider an internal cycle consisting of $2 k$ dipaths $k$ between $b_{i}$ and $c_{i}$ and $k$ between $b_{i}$ and $c_{i-1}$ (the indices are taken modulo $\mathrm{k}$ ). So the $b_{i}, i=1,2, \ldots, k$, have indegree 0 in the cycle and the $c_{i}, i=1,2, \ldots, k$, have outdegree 0 in the cycle. As the cycle is internal, there exist $k$ vertices $a_{i}, i=1,2, \ldots, k$ joined to the $b_{i}$ and $\mathrm{k}$ vertices $d_{i}, i=1,2, \ldots, k$ to which are joined the $c_{i}$. The set $\mathscr{P}$ of dipaths are $a_{1}, b_{1}, c_{1} ; b_{1}, c_{1}, d_{1} ; a_{i}, b_{i}, c_{i-1}, d_{i-1}$ and $a_{i}, b_{i}, c_{i}, d_{i}$ for $i=1,2, \ldots, k$ and $a_{1}, b_{1}, c_{k}, d_{k}$. They form a cycle of odd length $2 k+1$ in the conflict graph and so $w=3$ (see Figure 5).

Main Theorem Let $G$ be a DAG. Then, for any family of dipaths $\mathscr{P}, w(G, \mathscr{P})=\pi(G, \mathscr{P})$ if and only if $G$ does not contain an internal cycle. 

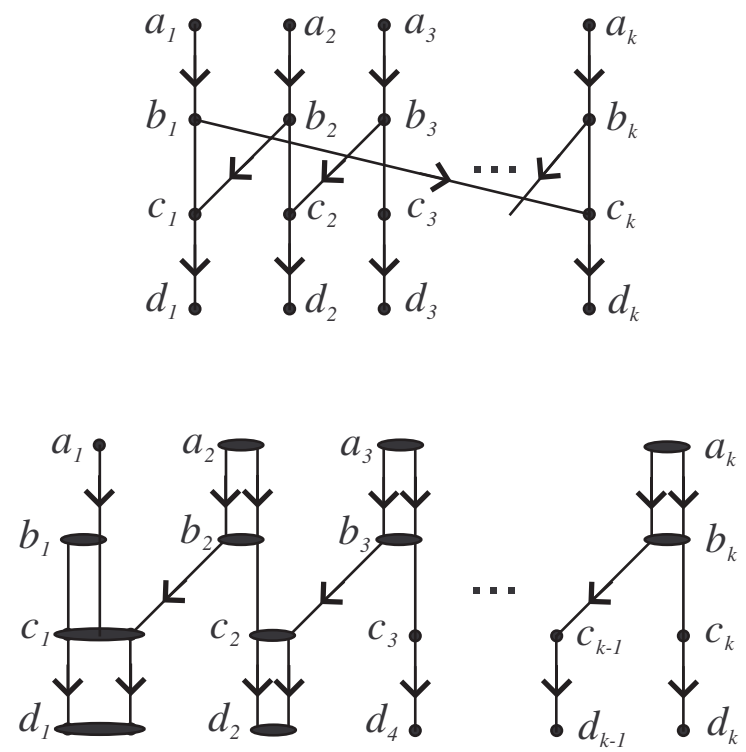

Figure 5. Internal cycle and family of dipaths with $\pi=2$ and $w=3$.

\section{UPP-DAGs}

First let us give some nice properties of the conflict graph associated to a family of dipaths of an UPP-DAG. We will first show that if $G$ is an UPP-DAG then $\pi$ is exactly the clique number of the conflict graph.

Property 3 If $G$ is an UPP-DAG then the dipaths in conflict have the following Helly property : if a set of dipaths are pairwise in conflict, then their intersection is a dipath.

Proof: If two dipaths intersect, then their intersection is a dipath. Indeed suppose the intersection contains two different dipaths $\left(x_{1}, y_{1}\right)$ and $\left(x_{2}, y_{2}\right)$ in this order. Then between $y_{1}$ and $x_{2}$ there are two dipaths, one via $P_{1}$ and the other via $P_{2}$ (see Figure 6 a).

So suppose $P_{1}$ and $P_{2}$ intersect in only one interval $\left(x_{1}, y_{1}\right)$, and $P_{3}$ intersects $P_{1}$ in an arc disjoint interval $\left(u_{1}, v_{1}\right)$. W.l.o.g. we may assume that $v_{1}$ is before $x_{1}$. Let $P_{3}$ intersects $P_{2}$ in the interval $\left(u_{2}, v_{2}\right)$.

Case $1: v_{2}$ is before $u_{1} . v_{2}$ cannot be after $y_{1}$ on $P_{2}$ otherwise there will be a directed cycle. So $v_{2}$ is before $x_{1}$ on $P_{2}$ and we have two dipaths from $v_{2}$ to $x_{1}$, one via $P_{2}$ and the other one via $P_{3}$ till $u_{1}$ and then via $P_{1}$ (see Figure $6 \mathrm{~b})$.

Case 2: $u_{2}$ is after $v_{1}$ on $P_{3}$ If $u_{2}$ is before $x_{1}$ on $P_{2}$ we have two dipaths from $v_{1}$ to $x_{1}$ one via $P_{1}$ and the other

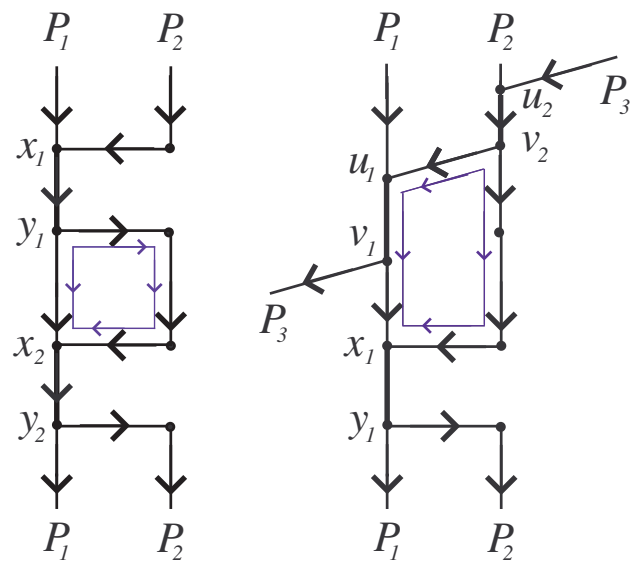

a)

b)

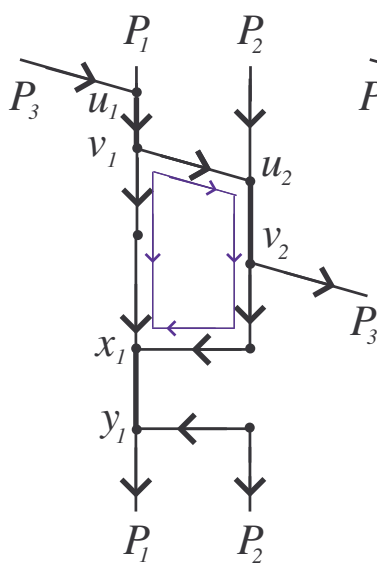

c)

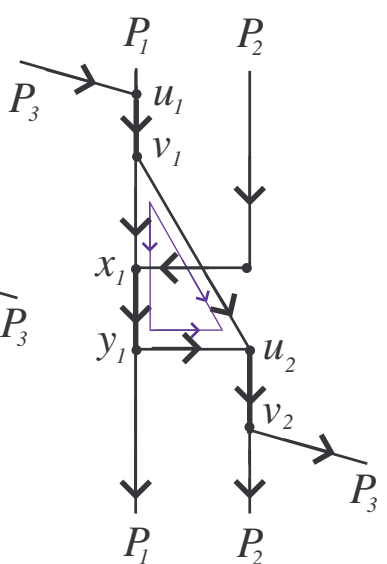

d)

\section{Figure 6. Helly property}

going from $v_{1}$ to $u_{2}$ via $P_{3}$ and to $x_{1}$ via $P_{2}$. If $u_{2}$ is after $y_{1}$, we have two dipaths from $v_{1}$ to $u_{2}$ one via $P_{3}$ and the other via $P_{1}$ till $y_{1}$ and $P_{2}$ (see Figure $6 \mathrm{c}$ and d).

Lemma 4 (Crossing lemma) Let $G$ be an UPP-DAG and let $P_{1}$ and $P_{2}$ be two disjoint dipaths. Consider $Q_{1}$ and $Q_{2}$ two disjoint dipaths intersecting $P_{1}$ and $P_{2}$. If $Q_{1}$ intersects $P_{1}$ before $Q_{2}$, then $Q_{2}$ intersects $P_{2}$ before $Q_{1}$.

Proof: Suppose that $Q_{2}$ intersects $P_{2}$ after $Q_{1}$. Two cases can happen according $Q_{2}$ intersects first $P_{1}$ (Figure 7 a) or $P_{2}$ (Figure $7 \mathrm{~b}$ ). In both cases we get a contradiction; indeed in case a) we have two dipaths between $y_{1}$ and $x_{2}^{\prime}$ and in case b) two dipaths between $y_{1}$ and $y_{1}^{\prime}$. 


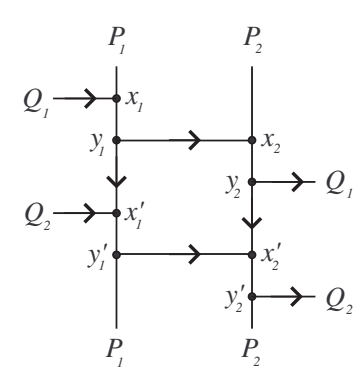

a)

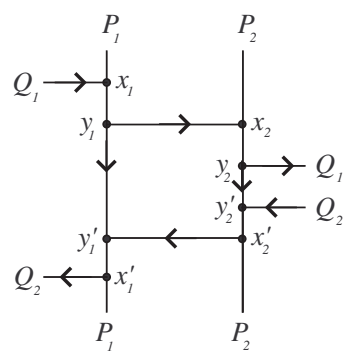

b)
Figure 7. Crossing lemma impossibility

Figure 8 shows the only possibility for the 4 dipaths, the conflict graph associated being a $C_{4}$.

Corollary 5 Let $G$ be an UPP-DAG. Then its conflict graph cannot contain a $K_{2,3}$.

Proof: Let $P_{1} P_{2}$ and $P_{3}$ be two disjoint dipaths. Consider $Q_{1}$ and $Q_{2}$ two disjoint dipaths, such that $Q_{i}$ intersects $P_{j}$. W.l.o.g.; suppose that $Q_{1}$ intersects $P_{1}$ before $Q_{2}$; then $Q_{2}$ intersects $P_{2}$ before $Q_{1}$ and $Q_{2}$ intersects $P_{3}$ before $Q_{1}$ a contradiction with the lemma applied to $Q_{1}, Q_{2}$ and $P_{2}$ and $P_{3}$.

We can similarly prove that if $G$ is an UPP-DAG then its conflict graph cannot contain a $K_{5}$ minus two independent edges.

In the preceding section we have seen that if $G$ is an UPP-DAG without internal cycle then for any family of admissible requests $w=\pi$; indeed the UPP property implies that each request can be routed via a unique dipath.

Now we will study UPP-DAGs with internal cycles. We still do not know if the number of wavelengths is bounded with respect to the load. We strongly conjecture that the ratio is unbounded. However we will show that for UPPDAGs with only one internal cycle there is a tight bound.

Theorem 6 Let $G$ be an UPP-DAG with only one internal cycle. Then for any family of dipaths $\mathscr{P}$,

$$
w(G, \mathscr{P}) \leq\left\lceil\frac{4}{3} \pi(G, \mathscr{P})\right\rceil
$$

Proof: Call $C$ the unique internal cycle of $G$. Let $(a, b)$ be an $\operatorname{arc}$ of $C$ with maximal load (upon the $\operatorname{arcs}$ of $C$ ). Define:

- $A_{a}$ to be the set of vertices $v$ of $G$ such that there is a dipath from $v$ to $a$

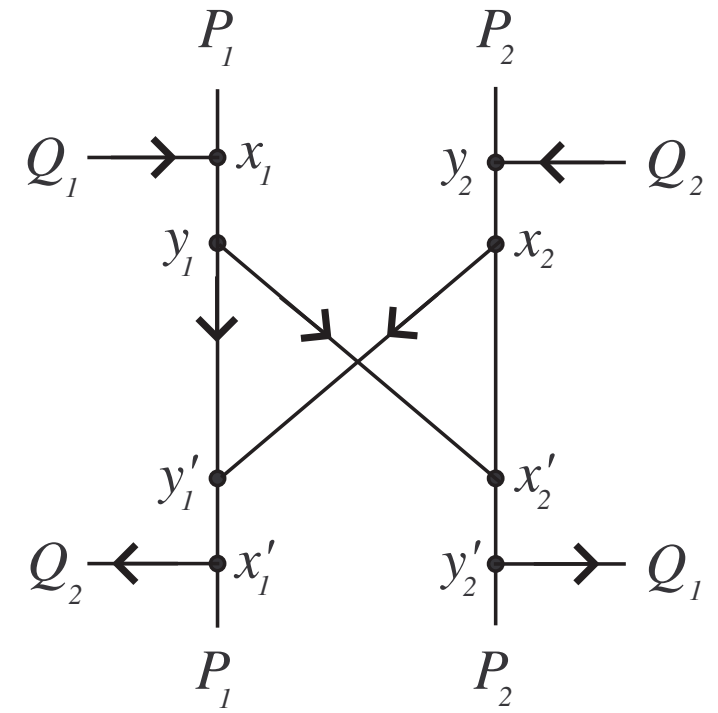

Figure 8. Crossing lemma possibility

- $S_{b}$ to be the set of vertices $v$ of $\mathrm{G}$ such that there is a dipath from $b$ to $v$.

Remark that $A_{a} \cap S_{b}=\emptyset$.

The idea of the proof is first to build an UPP-DAG $\tilde{G}$ without internal cycle, then to apply the result of section 3 to obtain an admissible coloring, which will be updated by introducing new colors in order to obtain a valid coloring for $G$.

Let $\tilde{G}$ be an UPP-DAG built from $G$ by replacing the arc $(a, b)$ with two $\operatorname{arcs}(\mathrm{a}, \mathrm{s})$ and $(\mathrm{t}, \mathrm{b})$. The family of dipaths $\mathscr{P}$ is replaced by $\tilde{\mathscr{P}}$ where all the dipaths are the same except for those which have their initial vertex $x$ in $A_{a}$ and their terminal vertex $y$ in $S_{b}$. Such a dipath [xy] is replaced in $\tilde{\mathscr{P}}$ by two dipaths one [xs] from $x$ to $s$ and the other [ty] from $t$ to $y$. Remark that $\tilde{G}$ has no internal cycle, that the load of $(a, s)$ and $(t, b)$ is equal to the load of $(a, b)$ and that the load of any other arc is unchanged. Hence we can apply theorem 1 to $\tilde{G}$ and obtain a coloring with $w(\tilde{G}, \tilde{\mathscr{P}})=\pi(\tilde{G}, \tilde{\mathscr{P}})$.

If $\pi(a, b)<\pi(G, \mathscr{P})$ we can consider a new set of dipaths obtained by adding $\pi(G, \mathscr{P})-\pi(a, b)$ copies of $[a, b]$ to the set of dipaths $\mathscr{P}$. Remark that if we have a coloring of $\left(G, \mathscr{P}^{\prime}\right)$ with $\omega^{\prime}\left(G, \mathscr{P}^{\prime}\right)$ colors then $\omega(G, \mathscr{P})<\omega^{\prime}\left(G, \mathscr{P}^{\prime}\right)$. Hence in the remaining we assume that $\pi(a, b)=\pi(G, \mathscr{P})$. Call this load $\pi$ and let $\left.\left\{\left[x_{0} y_{0}\right], \ldots,\left[x_{\pi-1} y_{\pi-1}\right]\right\}\right]$ be the family of the dipaths containing $(a, b)$. It is replaced in $\tilde{\mathscr{P}}$ by the two families: $\left\{\left[x_{0} s\right], \ldots,\left[x_{\pi-1} s\right]\right\}$ and $\left\{\left[t y_{0}\right], \ldots,\left[t y_{\pi-1}\right]\right\}$. These dipaths can be colored using $\pi$ colors since $\tilde{G}$ has no internal cycle. 
Now we will build a valid coloring of $(G, \mathscr{P})$.

Call $\tilde{\mathscr{P}}\left(A_{a}, s\right)$ the subset of dipaths starting from a vertex of $A_{a}$ to $s$ and $\tilde{\mathscr{P}}\left(t, S_{b}\right)$ the subset of dipaths starting from $t$ to a vertex of $S_{b}$ and let $\mathscr{D}=\tilde{\mathscr{P}}\left(A_{a}, s\right) \cup \tilde{\mathscr{P}}\left(t, S_{b}\right)$.

The proof relies on the following facts:

- Fact 1: If $P$ is a dipath of $\tilde{\mathscr{P}}$ not in $\mathscr{D}$ (so a dipath of $G$ too), then it intersects at most one dipath of $\mathscr{D}$. Indeed suppose $P$ intersect a dipath $\left[x_{i} s\right]$ in $\left[u_{i} v_{i}\right]$ and then another dipath $\left[x_{j} s\right]$ in $\left[u_{j} v_{j}\right]$.Then we get two dipaths from $v_{i}$ to $s$, one via $\left[x_{i} s\right]$ and the other via $P$ and then $\left[v_{j} s\right]$ contradicting the UPP property. The cases where $P$ intersect two dipaths of $\tilde{\mathscr{P}}\left(t, S_{b}\right)$ or one in $\tilde{\mathscr{P}}\left(A_{a}, s\right)$ and one in $\tilde{\mathscr{P}}\left(t, S_{b}\right)$ can be proven in a similar way.

- Fact 2: Suppose there exist two dipaths $P$ and $Q$ of $\tilde{\mathscr{P}}$ not in $\mathscr{D}$ such that $P$ intersects a dipath of $\mathscr{D}$ and $Q$ intersects another dipath of $\mathscr{D}$, then $P$ and $Q$ cannot intersect. There are different cases according to the kind of dipaths they intersect, but in all cases we get a contradiction by finding either a directed cycle or two dipaths between some pair of vertices or an internal cycle in $\tilde{G}$. As example of one possible case, suppose $P$ intersects $Q$ in $[u v]$ and then a dipath $\left[t y_{i}\right]$ in $\left[u_{i} v_{i}\right]$ and $\mathrm{Q}$ intersects after $P$ a dipath $\left[t y_{j}\right]$ in $\left[u_{j} v_{j}\right]$; then we have an internal cycle consisting of the dipath between $v$ and $u_{i}$ on $P$, followed by the reverse dipath from $u_{i}$ to $t$ on $\left[t y_{i}\right]$, then the dipath from $t$ to $u_{j}$ on $\left[t y_{j}\right]$, and the reverse dipath from $u_{j}$ to $v$ on $Q$.

We can subdivide the set of colors as follows:

We define $C_{1}$ as the set of colors $\alpha$, such that there exists an index $k$, such that the dipaths $\left[x_{k} s\right]$ and $\left[t y_{k}\right]$ have both the color $\alpha$. In that case we will color the dipath $\left[x_{k} y_{k}\right]$ with color $\alpha$.

We define $C_{2}$ as the set of pairs of colors $\alpha$ and $\beta$, such that there exist indices $i$ and $j$ such that $\left[x_{i} s\right]$ and $\left[t y_{j}\right]$ have both the color $\alpha$ and $\left[x_{j} s\right]$ and $\left[t y_{i}\right]$ have both the color $\beta$.

More generally we define $C_{p}$ as the set of p-tuples of colors $\alpha_{j}, j=1,2, \ldots, p$, such that there exist $p$ indices $i_{j}$, $j=1,2, \ldots, p$, such that the $\left[x_{i_{j}} s\right]$ have color $\alpha_{j}$ and $\left[t y_{i_{j}}\right]$ have color $\alpha_{j+1}$ (indices taken modulo $p$ ).

Therefore $\pi=\left|C_{1}\right|+2\left|C_{2}\right|+\ldots+p\left|C_{p}\right|+\ldots$.

Now we will prove that using an extra color for each ptuple of $C_{p}$, for $p>2$, we can obtain a valid coloring for G.

So consider, for $p>2$, a p-tuple of colors in $C_{p}, \alpha_{j}$, $j=1,2, \ldots, p$, and the $p$ indices $i_{j}, j=1,2, \ldots, p$, such that $\left[x_{i_{j}} s\right]$ have color $\alpha_{j}$ and $\left[t y_{i_{j}}\right]$ have color $\alpha_{j+1}$ (indices taken modulo $p$ ). We color in $G$ the dipath $\left[x_{i_{1}} y_{i_{1}}\right]$ with a new color $\gamma$ and, for $j \geq 2$, the dipaths $\left[x_{i_{j}} y_{i_{j}}\right]$ with color $\alpha_{j}$. Doing so all these dipaths have different colors. It might happen that one dipath $P_{j}$ with color $\alpha_{j}$ intersects now $\left[t_{i_{j}}\right]$ (previously colored with $\alpha_{j+1}$ ); in that case this dipath is unique by fact 1 and we recolor it with the extra color $\gamma$. All these dipaths recolored $\gamma$ cannot intersect $\left[x_{i_{1}} y_{i_{1}}\right]$ by Fact 1 and cannot intersect pairwise by Fact 2 .
So all the dipaths colored $\gamma$ are pairwise disjoint.

At this stage of the proof we have used one extra color for each p-tuple of colors in $C_{p}$, with $p>2$ and therefore we have altogether $w=\left|C_{1}\right|+3\left|C_{2}\right|+\ldots+(p+1)\left|C_{p}\right|+\ldots$ colors giving a bound of $\frac{3 \pi}{2}$ colors.

To get the value $\frac{4 \pi}{3}$ we have to pay more attention to the colors of $C_{2}$.

Suppose we can find a pair of elements of $C_{2}, \alpha_{j}, \beta_{j}$, $j=1,2$ and indices $i_{2 j-1}, i_{2 j}, j=1,2$ such that the dipaths $\left[x_{i_{2 j-1}} s\right]$ and $\left[t y_{i_{2 j}}\right]$ have both color $\alpha_{j}$ and $\left[x_{i_{2 j}} s\right]$ and $\left[t y_{i_{2 j-1}}\right]$ have both color $\beta_{j}$. Then we color the 4 corresponding dipaths in $G$ as follows: $\left[x_{i_{1}} y_{i_{1}}\right]$ with a new color $\gamma ;\left[x_{i_{2}} y_{i_{2}}\right]$ with color $\beta_{1} ;\left[x_{i_{3}} y_{i_{3}}\right]$ with color $\alpha_{2} ;\left[x_{i_{4}} y_{i_{4}}\right]$ with color $\beta_{2}$.

All these dipaths have therefore distinct colors. It might happen that another (unique) dipath $P$ with color $\beta_{1}$ (resp. $\alpha_{2}$, resp. $\beta_{2}$ ) intersects $\left[t y_{i_{2}}\right]$ (resp. $\left[t y_{i_{3}}\right]$, resp $\left[t y_{i_{4}}\right]$. Recolor these dipaths with color $\gamma$. By Facts 1 and 2 all the dipaths colored $\gamma$ are pairwise disjoint. So we need only 5 (and not 6 as previously) colors to deal with the 4 dipaths.

We do this recoloring as soon as we can find distinct pairs of elements in $C_{2}$. So if $\left|C_{2}\right|=2 h+r$, with $r=0$ or 1 we need to recolor the dipaths with colors in $C_{2}, 5 h+2 r$ colors a number always $\leq \frac{8}{3}\left|C_{2}\right|$ if $h \geq 1$.

So, if $\left|C_{2}\right| \geq 2$ or $\left|C_{2}\right|=0$, we are able to color $G$ with $w=\left|C_{1}\right|+\frac{8}{3}\left|C_{2}\right|+4\left|C_{3}\right|+\ldots+(p+1)\left|C_{p}\right|+\ldots$ colors, a number always less than or equal to $\left\lceil\frac{4}{3} \pi(G, \mathscr{P})\right\rceil$.

It remains to deal with $\left|C_{2}\right|=1$; if, all $C_{p}$ are empty for $p \geq 2$, we color $G$ with $w=\left|C_{1}\right|+3 \leq \frac{4}{3}\left(\left|C_{1}\right|+2\right)=$ $\frac{4}{3} \pi$ colors. Otherwise consider for some $p>2$, a p-tuple of colors in $C_{p}, \alpha_{j}, j=1,2, \ldots, p$, and the $p$ indices $i_{j}$, $j=1,2, \ldots, p$, such that the $\left[x_{i_{j}} s\right]$ have color $\alpha_{j}$ and $\left[t y_{i_{j}}\right]$ have color $\alpha_{j+1}$ (indices taken modulo $p$ ). We color in $G$ the dipath $\left[x_{i_{1}} y_{i_{1}}\right]$ with a new color $\gamma$ and, for $j \geq 2$, the dipaths $\left[x_{i_{j}} y_{i_{j}}\right]$ with color $\alpha_{j}$. Let $\alpha$ and $\beta$ be the colors of the unique element of $C_{2}$ and let the indices $i_{p+1}$ and $i_{p+2}$ be such that $\left[x_{i_{p+1}} s\right]$ and $\left[t y_{i_{p+2}}\right]$ have both the color $\alpha$ and $\left[x_{i_{p+2}} s\right]$ and $\left[t y_{i_{p+1}}\right]$ have both the color $\beta$. rRcolor $\left[x_{i_{p+1}} y_{i_{p+1}}\right]$ with color $\alpha$ and $\left[x_{i_{p+2}} y_{i_{p+2}}\right]$ with color $\alpha_{1}$. So we use only an extra color for the dipaths associated to the colors in the $C_{2}$ and $C_{p}$. Altogether we have $w=\left|C_{1}\right|+$ $2+4\left|C_{3}\right|+\ldots+(p+1)\left|C_{p}\right|+\ldots$ a number always less than or equal to $\left\lceil\frac{4}{3} \pi\right\rceil$ as $\pi=\left|C_{1}\right|+1+3\left|C_{3}\right|+\ldots+p\left|C_{p}\right|+\ldots$.

The argument of the proof can be repeated in the case of more than one cycle. This leads to a bound of $\left[\frac{4}{3}^{C} \pi(G, \mathscr{P})\right]$ if $C$ is the number of internal cycles of the UPP-graphs.

To show that the bound of the theorem is tight we have to exhibit a family of dipaths reaching the bound. The example given in Theorem 2 shows that the bound is tight for $\pi=2$. 
Indeed, for $k=2$, we have a graph $G$ and a set of 5 dipaths such that the conflict graph is a $C_{5}$ and therefore $w=3$ Replacing each of these dipaths with $h$ identical dipaths we obtain a family of $5 h$ dipaths with $\pi=2 h$ and $w=\left\lceil\frac{5 h}{2}\right\rceil$ giving a ratio of $\frac{5}{4}$ which does not reach the bound.

Theorem 7 There exists an UPP-DAG G with one internal cycle and a family $\mathscr{P}$ of dipaths such that

$$
w(G, \mathscr{P})=\left\lceil\frac{4}{3} \pi(G, \mathscr{P})\right\rceil
$$
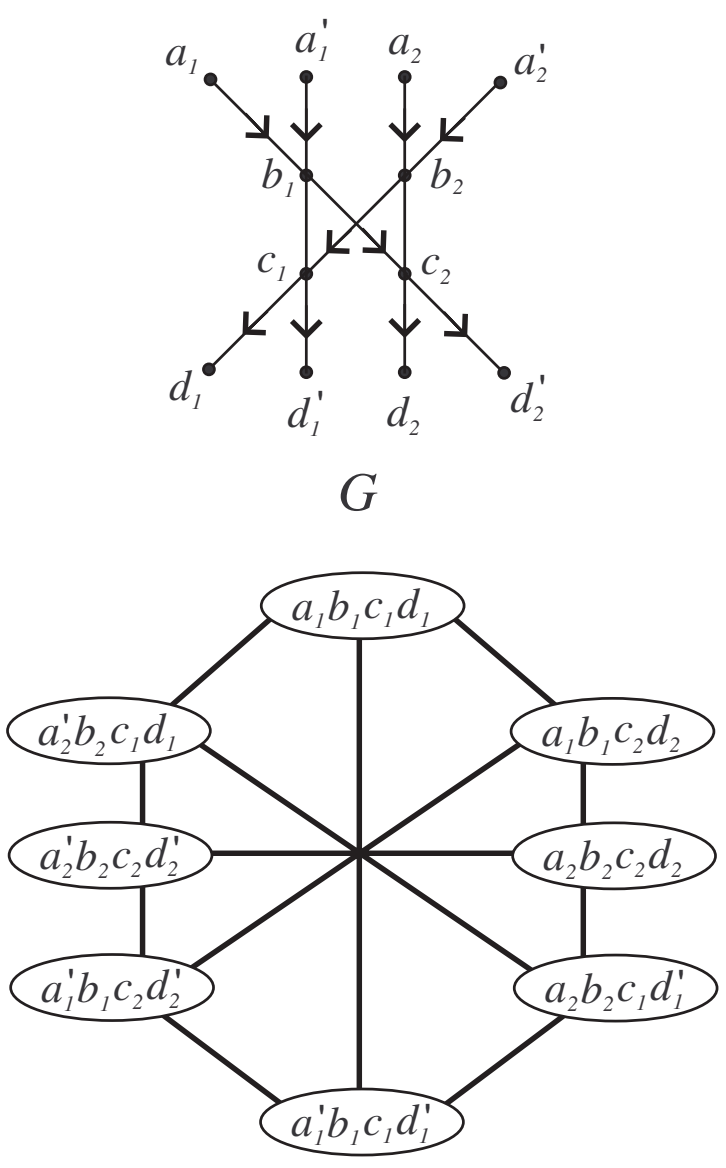

Conflict graph

Figure 9. Example for an UPP-DAG

Proof: The following example is due to Frédéric Havet (private communication). It consists of 8 dipaths generating the conflict graph consisting of a cycle of length 8 plus chords between the antipodal vertices. Here again $\pi=2$ and $w=3$; but if we replace each of these dipaths with $h$ identical dipaths we obtain a family $\mathscr{P}$ of $8 h$ dipaths with $\pi=2 h$ and $w=\left\lceil\frac{8 h}{3}\right\rceil$; indeed in the conflict graph an independent set has at most 3 vertices and so we need at least $\frac{8 h}{3}$ colors. Therefore this family satisfies the theorem.

\section{Concluding remarks}

In this article we have determined when a DAG $G$ satisfies $w(G, \mathscr{P})=\pi(G, \mathscr{P})$ for any family of dipaths $\mathscr{P}$. We have introduced an apparently new family of DAGS the UPP-DAGS. We believe that they have already been used in parallel computing but could not find any reference. Characterization and study of the properties of these digraphs is of interest in itself. We conjecture that there is no bouded ratio between the number of wavelengths and the load in presence of an unlimited number of cycles. It will be also interesting to find other digraphs which satisfy $w(G, \mathscr{P})=\pi(G, \mathscr{P})$ for any family $\mathscr{P}$ or for some specific families. Finally the techniques developed here can help to solve the problem which initially motivated this study that is to find for a given $w$ the maximum number of requests (or dipaths in UPP-DAGS) chosen among a given family of requests that can be satisfied (for example in the all to all case, ie one dipath for any possible pair of vertices). Our theorem shows that we have only to compute the load. The case of rooted trees appears already as a difficult one.

\section{Acknowledgments:}

This work has been partially funded by European projects IST FET AELOUS and has been done in the CRC CORSO with France Telecom.

We thank D. Coudert, L. Gargano, F. Havet, S. Pérennes, I. Sau-Valls, J-S. Sereni, U. Vaccaro and the referees for their helpful comments. We also thank the two brothers Kostas and Giorgos Kadarakis of Infinity Blue for their kindness and hospitality.

\section{References}

[1] B. Beauquier, J.-C. Bermond, L. Gargano, P. Hell, S. Pérennes, and U. Vaccaro. Graph problems arising from wavelength-routing in all-optical networks. In IEEE Workshop on Optics and Computer Science, Geneva, Switzerland, Apr. 1997.

[2] B. Beauquier, P. Hell, and S. Pérennes. Optimal wavelengthrouted multicasting. Discrete Applied Mathematics, 84:1520, 1998.

[3] J.-C. Bermond, L. Braud, and D. Coudert. Traffic grooming on the path. Theoretical Computer Science, 2007. To appear. 
[4] J.-C. Bermond, M. Cosnard, D. Coudert, and S. Pérennes. Optimal solution of the maximum all request path grooming problem. In Proceedings of the Advanced International Conference on Telecommunications, AICT06, Guadeloupe, France, February 2006.

[5] I. Caragiannis, C. Kaklamanis, and P. Persiano. Wavelength routing in all-optical tree networks: A survey. Computers and Artificial Intelligence, 2001.

[6] R. Dutta and N. Rouskas. A survey of virtual topology design algorithms for wavelength routed optical networks. $O p$ tical Networks Magazine, 1(1):73-89, 2000.

[7] R. Dutta and N. Rouskas. Traffic grooming in WDM networks: Past and future. IEEE Network, 16(6):46-56, 2002.

[8] T. Erlebach, K. Jansen, C. Kaklamanis, M. Mihail, and P. Persiano. Optimal wavelength routing on directed fiber trees. Theoretical Computer Science, 221(1-2):119-137, 1999.

[9] L. Gargano, P. Hell, and S. Prennes. Colouring all directed paths in a symmetric tree with an application to optical networks. Journal of Graph Theory, 38(4):183-196, 2001.

[10] J. Kleinberg and A. Kumar. Wavelength conversion in optical networks. In Proceedings of SODA'99, Baltimore,MD, USA, January 1999.

[11] B. Mukherjee. Optical communication networks. McGrawHill,New York, 1997.

[12] R. Ramaswami and K. Sivajaran. Routing and wavelength assignment in all-optical networks. IEEE Transactions on NetworkingNetwork, 3(5):489-500, 1995.

[13] R. Ramaswami and K. Sivajaran. Optical networks : a practical perspective. Morgan kaufmann, San Francisco, 1998. 